PSICOLOGIA

IBEROAMERICANA
Psicología Iberoamericana ISSN: 1405-0943

revista.psicologia@ibero.mx

Universidad Iberoamericana, Ciudad de México México

\title{
Efecto de la pandemia en hábitos de vida y salud mental: comparación entre dos universidades en México
}

Morfín Lopez, Teresita; Mancillas Bazán, Celia; Camacho Gutiérrez, Everardo; Polanco Hernández, Graciela; Hernández Vidrio, Mariana

Efecto de la pandemia en hábitos de vida y salud mental: comparación entre dos universidades en México

Psicología Iberoamericana, vol. 29, núm. 3, Esp., e293338, 2021

Universidad Iberoamericana, Ciudad de México, México

Disponible en: https://www.redalyc.org/articulo.oa?id=133968747004

DOI: https://doi.org/10.48102/pi.v29i3.338

\section{(c) (1)}

Esta obra está bajo una Licencia Creative Commons Atribución 4.0 Internacional. 
Psicología Iberoamericana, vol. 29, núm. 3, Esp., e293338, 2021

Universidad Iberoamericana, Ciudad de México, México

Recepción: 02 Febrero 2021 Aprobación: 22 Junio 2021

DOI: https://doi.org/10.48102/ pi.v29i3.338

Redalyc: https://www.redalyc.org/ articulo.oa?id=133968747004
Artículos

\section{Efecto de la pandemia en hábitos de vida y salud mental: comparación entre dos universidades en México}

Effect of the pandemic on living habits and mental health: A comparison between two universities in Mexico

Teresita Morfín Lopez teremor@iteso.mx Instituto Tecnológico y de Estudios Superiores de Occidente (ITESO), México

https://orcid.org/0000-0003-3994-3025 Celia Mancillas Bazán celia.mancillas@ibero.mx Universidad Iberoamericana, Ciudad de Méxic, México

iD https://orcid.org/0000-0002-6833-7959

Everardo Camacho Gutiérrez ecamacho@iteso.mx Instituto Tecnológico y de Estudios Superiores de Occidente (ITESO), México

https://orcid.org/0000-0002 85927897 Graciela Polanco Hernández graciela.polanco@ibero.mx Universidad Iberoamericana, Ciudad de México, México

https://orcid.org/0000-0003-1661-7518

Mariana Hernández Vidrio mahevi2292@gmail.com Instituto Tecnológico y de Estudios Superiores de Occidente (ITESO),

México

https://orcid.org/0000-0001-9467-4927

Resumen: El objetivo del estudio era analizar la valoración del impacto de la pandemia en hábitos de vida y salud mental, con participantes de dos universidades privadas, una en Ciudad de México y otra en Guadalajara. Se utilizó un diseño transversal cuantitativo con 246 personas de la Universidad de CDMX (177 mujeres y 68 hombres) y 382 personas (220 mujeres y 159 hombres) de Guadalajara, con edades entre 16 y 80 años, respondiendo el cuestionario PSY-COVID mediante invitación electrónica por correo o facebook,durante junio y julio del 2020. Se analizaron porcentajes de respuestas y se aplicó chi cuadrada para analizar las variables de género y edad y $\mathrm{r}$ de Pearson y prueba $T$ para hábitos y salud mental. Existe mayor valoración de afectación en jóvenes, y mujeres en ansiedad y depresión, y en calidad de sueño y actividad laboral. Las diferencias son significativas. Hay un deterioro en los hábitos en general, así como con respecto a ansiedad y depresión en ambos universitarios, aun cuando la diferencia no es significativa entre ellos. Jóvenes y mujeres son más vulnerables.

Palabras clave: ansiedad, depresión, actividad física, sueño, alimentación.

Abstract: The objective of the study was to analyse the assessment of the impact of the pandemic on lifestyle and mental health, with participants from two private universities, one in Mexico City and the other in Guadalajara. A quantitative cross-sectional design was implemented. Participants included 246 people from the University of CDMX (177 women and 68 men) and 382 people (220 women and 159 men) from Guadalajara, aged between 16 and 80 years old. Participants answered the PSY-COVID questionnaire via 
an email invitation or on Facebook, during June and July 2020. Percentages of responses were analysed. Chi-square was applied for gender and age and Pearson's $r$ and T-test for habits and mental health. Results revealed that there was a higher assessment of affectation in young people and women in anxiety and depression, and in quality of sleep and work activity. The differences were significant. There was a deterioration in lifestyle habits in general, as well as with respect to anxiety and depression in both university groups, even though the difference is not significant between them. Youth and women were found to be more vulnerable.

Keywords: anxiety, depression, physical activity, sleep, food.

\section{Introducción}

De acuerdo a la Organización Mundial de la Salud (OMS) (2021), el COVID\#19 es una enfermedad infecciosa causada por el coronavirus que se ha descubierto recientemente. La OMS caracterizó al COVID-19 como una pandemia que se ha esparcido en 213 países incluyendo México en donde se han detectado 4.9 millones de casos conocidos hasta el 19 de mayo del 2020 de acuerdo al reporte presentado por la OMS (Villaseñor Lopez et al., 2020). Esta enfermedad ha traído grandes cambios en el estilo de vida de las personas, como: los alimenticios, trastornos del sueño, ansiedad y depresión generados por el confinamiento, así como la adaptación a nuevas formas de trabajo, esto a su vez ha generado en las personas cierto grado de resiliencia, permitiéndoles sobrellevar la contingencia de salud (Organización Panamericana de la Salud, 2020).

La pandemia global por COVID-19 ha tenido efectos en diversos aspectos que conforman la vida de las personas, familias, comunidades y países. Las consecuencias son severas, como las 221, 080 defunciones al 21 de mayo del 2021, registradas en México y por el impacto en la economía y en el empleo, lo que representa una amenaza a la subsistencia como nos informa la Secretaría de Salud en su informe técnico diario a través de su página web (Gobierno de México, 2020). La "nueva normalidad", planteada como respuesta inmediata, resulta incierta y precaria.

Así, el foco principal de la OMS fue el de atender una crisis en la salud física, aunque es ahora reconocido, cada vez con mayor importancia, la comprensión de los efectos de la pandemia en la salud mental, por lo que resulta importante fortalecer investigaciones sobre el impacto de la pandemia tanto en la salud física como en la mental para apoyar la toma de decisiones con respecto a la salud. La OMS (1948), define la salud como un estado de bienestar físico, mental y social completo y no meramente la ausencia de enfermedad o padecimiento; así, la salud incluye el funcionamiento mental, físico y social, los cuales están relacionados entre sí y son interdependientes.

\section{Problemas de salud mental asociados a la pandemia}

El incremento de trastornos de ansiedad, depresión y estrés ha sido reportado en distintas investigaciones y revisiones de la literatura científica, en la población en general como se reportan estudios en China (Huang \& Zhao, 2020), Italia (Mazza et al., 2020) y Pakistán 
(Riaz et al., 2021). Las consecuencias psicológicas asociadas a la experiencia de la pandemia son múltiples, desde síntomas aislados hasta trastornos complejos, como estrés, miedo, ira, frustración, trastornos de ansiedad, trastornos de estrés postraumático, depresión, trastorno obsesivo compulsivo y violencia, entre otros (Ferrer, 2020; Sandín et al., 2020).

El distanciamiento físico y otras medidas relacionadas han sido la respuesta inmediata de salud pública, y a pesar de su evidente fortaleza para evitar el contagio del COVID-19, llevan el riesgo de aumentar el aislamiento, especialmente en poblaciones vulnerables. Las restricciones de la interacción social presencial característica del distanciamiento social, una de las formas de la "nueva normalidad", es un factor que, por un lado, es una fuente de estrés y por otro, ha aumentado la conciencia de la necesidad del contacto cercano en la vida familiar y social, esto es, la cercanía en los vínculos afectivos y su manifestación en los diversos aspectos de la vida íntima y social (Almagiá, 2004; Cálad, 2003; Marquina Lujan, 2020).

Ramírez-Ortiz et al. (2020), destacan que, en las personas con aislamiento social, con movilidad restringida y poco contacto con otros, aún en circunstancias normales, son propensas a desarrollar insomnio, ansiedad, depresión y trastorno de estrés postraumático. Las personas que están bajo estrés por el posible contagio pueden presentar una marcada ansiedad y una disminución significativa de la función social u ocupacional, lo que puede resultar en trastornos de adaptación y aún en trastornos de depresión mayor.

El confinamiento ha generado diversos padecimientos tanto en lo económico como en la salud. Otro efecto importante ha sido el trastorno del sueño como lo demuestra Di Renzo et al., (2020) en un estudio realizado en Italia, donde encontró que durante el confinamiento las personas encuestadas argumentaron dormir por periodos más prolongados. Un caso contrario al estudio realizado en México por Villaseñor Lopez et al., (2020) en el que las mujeres afirmaron dormir menos y despertar más durante la noche, a diferencia de los hombres. Otra afirmación de este hecho fue el estudio realizado en España por Pérez-Rodrigo et al., (2020) encontrando que el 37\% de los encuestados aseguraron tener problemas para dormir (Villaseñor Lopez et al., 2020). El descanso inadecuado afecta la calidad de vida de las personas provocando accidentes dentro y fuera del hogar a causa de una somnolencia excesiva, incluso volviéndolos más vulnerables a enfermedades como hipertensión, sobrepeso y obesidad (GuerreroZúñiga et al., 2018). El insomnio también es característico de los efectos propios de la enfermedad del Covid, empeorando el estado de salud y sistema inmunológico del paciente (Medina-Ortiz et al., 2020).

En un estudio realizado en Reino Unido, Proto y Quintana-Domeque (2021), evaluaron a la población antes y durante la pandemia. Utilizaron el Estudio Longitudinal de Hogares del Reino Unido y compararon los datos obtenidos con el mismo grupo de individuos, antes del COVID-19 (2017-2019) y durante la pandemia (abril 2020) y los cambios en salud 
mental fueron evaluados a través del GHQ-12 (Cuestionario General de Salud), entre los grupos étnicos en el Reino Unido. Encontraron que el aumento de la angustia varía según la etnia y el género. Atribuyen estos resultados a que la salud mental en la pandemia se ve afectada por las medidas de distanciamiento físico, que afecta diferencialmente a distintos grupos étnicos.

La ansiedad en alto grado puede hacer que las personas confundan dolores musculares benignos con dolores propios de la enfermedad, además de provocar pánico y comportamientos desadaptativos como el retraimiento social, compras compulsivas o de pánico, que puede afectar a la persona y a la comunidad, principalmente cuando se realizan gastos excesivos para el almacenamiento de recursos (Moreno-Proaño, 2020; Vivanco Vidal et al., 2020). La ansiedad y el estrés incrementa los niveles de cortisol lo cual inhibe el sistema inmunológico haciendo más proclive al organismo, para contraer alguna enfermedad por virus o bacterias (Segerstrom \& Miller, 2004).

Ammar et al. (2020), analizaron cuestionarios en países de Europa, Norte de África, Asia Occidental y América. Evaluaron los efectos del confinamiento en el bienestar emocional, el estado de ánimo y los sentimientos. Encontraron efectos significativos de la pandemia en la salud mental, especialmente en el bienestar psicológico, el estado de ánimo y los sentimientos. Se presentó menos bienestar durante el confinamiento, que antes del mismo, así como un aumento de síntomas depresivos.

En el mismo sentido, basándose en una revisión sistemática sobre síntomas de trastornos mentales durante la pandemia por COVID-19, Martínez Gómez et al. (2020) encontraron 16 artículos de estudios empíricos. La tendencia de los síntomas y trastornos más estudiados fueron ansiedad (28\%), depresión (23\%), estrés (15.3\%), trastorno del sueño-vigilia (9.6\%) y trastorno por estrés postraumático (5.7\%), asociados con el consumo de sustancias psicoactivas, bajo rendimiento académico, pérdida de trabajo, calidad del sueño, bajo autocuidado y baja percepción del estado de salud durante la pandemia.

La evaluación de problemas de la salud mental relacionados con el COVID -19 es uno de los aspectos que se reportan como prioritarios para lograr mediciones que permitan una comprensión de la magnitud del problema. Chandu et al. (2020), enfatizan la necesidad de desarrollar y validar herramientas psicométricas para evaluar los problemas de salud mental asociados al COVID-19.

Para Niederkrotenthaler et al. (2020), destacan la necesidad de llevar a cabo investigaciones a nivel mundial que permitan evaluar los efectos directos o indirectos del COVID-19.

Con base en esta información se cuestiona si existen diferencias entre las muestras de los participantes de las dos universidades estudiadas, con respecto a sus hábitos de vida y salud mental en las condiciones generadas por la pandemia. ¿Por género, edad, con respecto a hábitos de vida y su impacto en salud mental? ¿Existe correlación entre las variables medidas de hábitos de vida y salud mental? 
De tal forma, el objetivo del estudio es analizar el efecto psicológico en la población universitaria que se ha generado a partir de la propagación de la pandemia de COVID-19. Este efecto puede ser analizado primero, en los problemas psicológicos asociados con las medidas de restricción de la movilidad o de la enfermedad COVID-19. En segundo término, en los factores protectores de la salud mental y los cambios en los hábitos de vida, que se han experimentado durante el continuo al que se ha visto sometida la población entre el confinamiento y el desconfinamiento. Específicamente, se analizará comparativamente el impacto psicológico en dos poblaciones: en una Universidad privada de la Ciudad de México comparada con una Universidad privada de Guadalajara, para desarrollar estrategias preventivas y de intervención específicas para la atención en la salud mental de estos grupos. Se considera relevante identificar como las diferencias tanto de contexto físico (cantidad de población diferente entre ambas ciudades) como de contexto sociocultural (prácticas diferenciadas en función del grupo social característico de cada ciudad) impactan en la manera en que los universitarios afrontan las demandas y restricciones generadas por la pandemia.

\section{Método}

\section{Antecedentes}

Este trabajo forma parte de un proyecto de colaboración internacional denominado PSY-COVID coordinado por el Dr. Antoni Sanz de la Universidad Autónoma de Barcelona (López Fernández \& Sanz, 2020). En el estudio general participaron 40 universidades y más de 80 investigadores-colaboradores de 18 países de América y Europa.

\section{Objetivos de investigación}

La intención del trabajo de investigación es describir el impacto de la pandemia y sus restricciones en los participantes de ambas universidades, tanto en sus hábitos de vida como en su salud mental.

En segunda instancia, se considera que existirán semejanzas y diferencias con respecto a las variables evaluadas mediante el cuestionario. $\mathrm{Al}$ respecto de las diferencias, se hipotetiza que tanto las diferencias de contexto físico (como las diferencias en cantidad de población y lo que implica en tráfico, por ejemplo), así como el contexto cultural distinto (las prácticas grupales características de los habitantes una ciudad) pudieran explicarlas.

En el mismo tenor se analizará si existen diferencias o semejanzas por género y edad en cada uno de los dos grupos comparados, así como la covariación entre las variables evaluadas con objeto de identificar como dicha covariación conforma un grado de calidad de vida específico y diferenciado. 


\section{Participantes}

Los participantes fueron reclutados a través de materiales impresos y en línea distribuidos por centros académicos en México, así como de boca a boca mediante un muestreo por conveniencia. El instrumento fue completado en español. Todos los participantes otorgaron su consentimiento informado mediante un formulario en línea y respondiendo de manera anónima y se les informó que podrían abandonar el cuestionario en cualquier momento. Los criterios de inclusión fueron: tener 16 años o más (con consentimiento de los padres para los menores de 18 años) y residir en México al momento de completar la encuesta. La muestra de este estudio estuvo conformada por académicos, administrativos y estudiantes de dos universidades privadas: una de la Ciudad de México y otra situada en la ciudad de Guadalajara, Jalisco, México. En la universidad de la ciudad de Guadalajara, respondieron de forma anónima 382 participantes y en la universidad de la Ciudad de México, 246 participantes. 
Tabla 1

Tabla 1

Características demográficas de los participantes

Universidad de

Cd. Guadalajara
Universidad de

CDMX

\begin{tabular}{ccccccc} 
Variable & Categoría & Frecuencia & Porcentaje & Categoría & Frecuencia & Porcentaje \\
\hline Género & Masculino & 159 & 41.6 & Masculino & 68 & 27.6 \\
& Femenino & 220 & 57.6 & Femenino & 177 & 72 \\
& Otro & 3 & 0.8 & Otro & 1 & 0.4 \\
\hline Grupo & $16-26$ & 19 & 5 & $16-26$ & 49 & 19.9 \\
de edad & $27-37$ & 89 & 23.3 & $27-37$ & 68 & 27.6 \\
& $38-48$ & 132 & 34.6 & $38-48$ & 64 & 26 \\
& $49-59$ & 106 & 27.7 & $49-59$ & 44 & 17.9 \\
& $60-70$ & 29 & 7.6 & $60-70$ & 19 & 7.7 \\
& $71-80$ & 7 & 1.8 & $71-80$ & 2 & 0.8 \\
& & & & & & \\
\hline Nivel de & Básicos & 11 & 2.9 & Básicos & 4 & 1.6 \\
estudios & Universitarios & 371 & 97.1 & Universitarios & 242 & 98.4 \\
\hline Nivel de & Bajo & 22 & 5.8 & Bajo & 29 & 11.8 \\
ingresos & Medio & 284 & 74.3 & Medio & 175 & 71.1 \\
& Alto & 76 & 19.9 & Alto & 42 & 17.1
\end{tabular}

\section{Instrumentos}

Se aplicó el cuestionario diseñado para México con 122 ítems con opciones con base en escala Likert que evaluó diferentes dimensiones como son: características demográficas, hábitos de vida, salud mental, adaptación a la pandemia.

\section{Características demográficas}

Se recopiló información sobre: edad, género, educación, y nivel de ingresos. 


\section{Impactos relacionados con COVID en los hábitos de vida}

La información consideró cambios en diferentes aspectos de la vida cotidiana relacionados con la pandemia de COVID-19, que incluyen: actividad física, sueño, alimentación, ingresos, trabajo, relación con la pareja, relaciones con amigos y relaciones con vecinos. Los reactivos se calificaron en una escala Likert de 5 puntos $(1=$ mucho peor; $2=$ algo peor; 3 = igual; 4 = algo mejor; $5=$ mucho mejor).

\section{Salud mental}

Se utilizó el Patient Health Questionnaire-4 (PHQ-4; Löwe et al., 2010), el cual incluye dos preguntas para evaluar síntomas de depresión y dos preguntas sobre síntomas de ansiedad.

\section{Depresión}

Se utilizó el Cuestionario de Salud del Paciente (PHQ-2) (Kroenke et al., 2003). El instrumento contiene dos ítems en una escala de Likert de 4 puntos que fluctúa de 0 (ningún día) a 3 (casi cada día). El enunciado fue, "Ha experimentado: a) Poco interés o placer en hacer cosas, b) Decaimiento, depresión o desesperanza”. La escala presenta una consistencia interna adecuada $(\alpha=.77)$. La puntuación total obtenida en el PHQ-2 va de 0 a 6 y las puntuaciones iguales o superiores a 3 indican presencia de sintomatología depresiva (Löwe et al., 2010).

\section{Ansiedad}

Los síntomas de ansiedad en la población general se evaluaron con la Escala de Trastornos de Ansiedad Generalizada-2 (GAD-2) de Kroenke et al. (2007). Ésta presenta una adecuada consistencia interna en la población mexicana $(\alpha=.78)$. Se compone de dos ítems en una escala tipo Likert de 4 puntos que va de 0 (ningún día) a 3 (casi cada día). El enunciado general fue, "Ha experimentado: a) Nerviosismo o tensión, b) Incapacidad de dejar de preocuparse o de controlar la preocupación". Puntuaciones iguales o superiores a 3 de un rango de 0 a 6 indican la presencia de sintomatología ansiosa (Kroenke et al., 2003).

\section{Somatización}

Se utilizaron cinco preguntas del Patient Health Questionnaire-15 (PHQ-15; Kroenke et al., 2003), basadas en las recomendaciones para evaluar los problemas de somatización derivadas de una revisión sistemática (Zijlema et al., 2013). El rango de puntuación es de 0 - 15, donde las puntuaciones iguales o superiores a 5 indican presencia de síntomas somáticos. Los síntomas evaluados fueron: (a) dolor de cabeza, 
(b) náusea o dolor de estómago, (c) sensación de falta de aire, (d) mareo, y (e) dolor de espalda. Todos los ítems fueron calificados en una escala de frecuencia de 4 puntos con respecto a las últimas 2 semanas, que va de 0 (ningún día) a 3 (casi cada día).

\section{Diseño}

El estudio se desarrolló con un diseño transversal con enfoque cuantitativo de una sola etapa, basado en la administración del cuestionario online anónimo del estudio internacional PSY-COVID difundido en México. La encuesta fue revisada por un panel de 30 expertos en psicología de la salud, se inició el 4 de junio y finalizó el 31 de julio de 2020. El estudio fue aprobado por el Comité Ético de Experimentación Animal y Humana de la Universidad Autónoma de Barcelona y del Comité de ética de la Universidad situada en Guadalajara Se administró en una plataforma de encuestas en línea, fue difundido a través de las principales redes sociales y por correo electrónico.

\section{Procedimiento}

El equipo de investigadores del Nodo México tomó la decisión de excluir a quienes completaron el cuestionario después de Julio. El cuestionario fue difundido en México durante un período, entre junio y julio 2020, con la pretensión de realizar la aplicación en el momento de desescalada de las principales medidas gubernamentales de restricción de movilidad (confinamiento).

\section{Contexto del estudio}

Es importante caracterizar que ambas Universidades son privadas y con orientación religiosa. La Universidad situada en la Ciudad de México, se encuentra situada en una zona a la orilla de la Ciudad, lo que implica para la mayoría de los participantes, el invertir una buena cantidad de tiempo en desplazarse. A diferencia de la Universidad en Guadalajara, que aun cuando el campus se encuentra en un extremo de la ciudad, los tiempos de desplazamiento son mucho menores y muchos de sus asistentes acostumbran regresar a casa a comer y posteriormente volver a ir por la tarde a la Universidad.

Las cuotas universitarias en la Universidad de la CDMX son costosas y por lo tanto el tipo de estudiantes que asisten son de alto poder adquisitivo, a diferencia de la Universidad en la ciudad de Guadalajara, donde existe un alto porcentaje de becas y financiamiento que posibilita que el tipo de estudiantes que asiste sea de nivel socioeconómico medio. En la Universidad de la CDMX se tiene un ambiente más plural en el sentido de que una gran mayoría de su población es de origen foráneo a la ciudad (como caracteriza a cualquier capital), en el caso de la Universidad 
en Guadalajara, solamente el $30 \%$ de los estudiantes son foráneos y corresponden al noroeste del país.

Se considera que estas diferencias entre los participantes del estudio pueden resultar en formas diferentes de valorar la afectación de la pandemia en los hábitos de vida y la salud mental.

\section{Análisis de datos}

Se obtuvieron porcentajes de distribución de las respuestas de las muestras estudiadas para obtener una descripción comparativa entre los participantes de ambas instituciones de las diferentes variables evaluadas.

Se hizo un análisis de chi-cuadrada para identificar y reportar si existen o no diferencias significativas en las variables de género y edad, así como la prueba $T$ en las variables tanto de hábitos de vida como de salud mental de las muestras analizadas y posteriormente se hizo un análisis correlacional de Pearson entre algunas de las variables estudiadas, previo análisis de la distribución normal de algunas de las variables. Para procesar los datos se utilizó el programa SPSS versión 26.

\section{Resultados}

A continuación, se muestra un conjunto con 7 figuras que representan el porcentaje de opciones para ambas universidades. La Universidad de la CDMX y la Universidad de la Ciudad de Guadalajara.

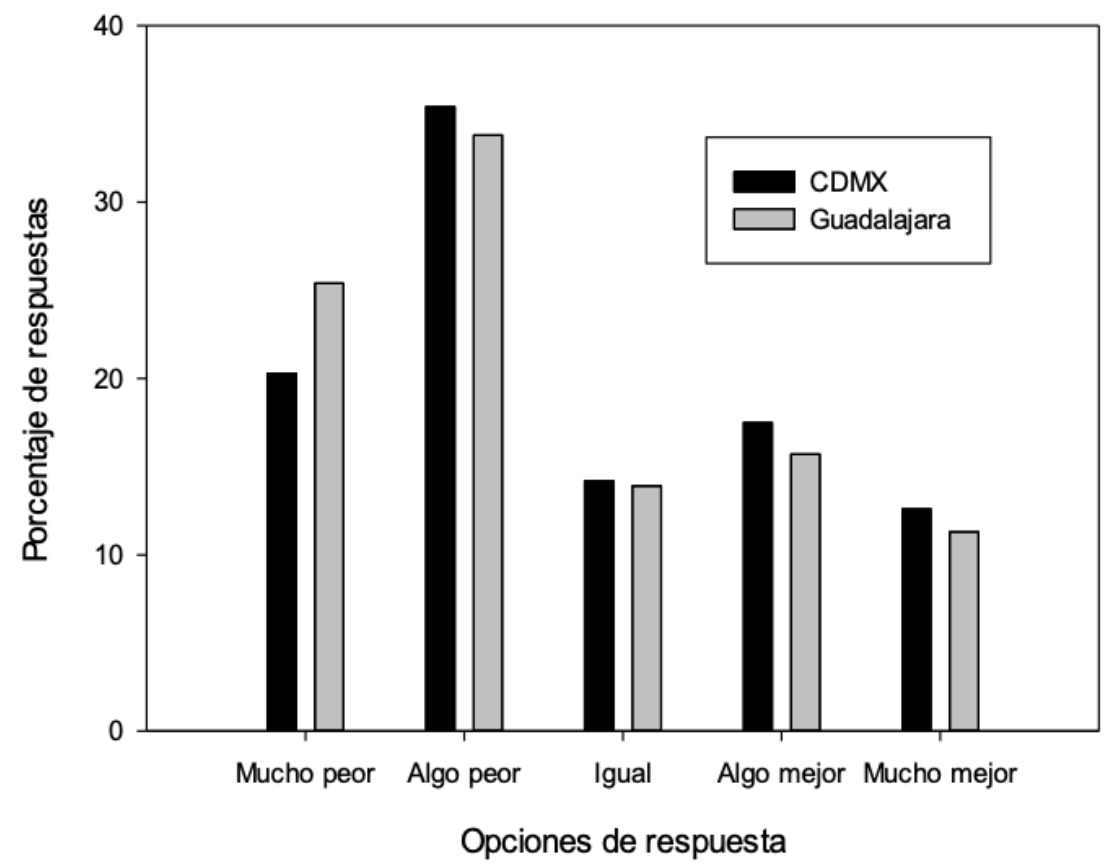

Figura 1.- Valoración de la actividad física

Figura 1 
En la Figura 1 se ilustra que hay una valoración de la actividad física mucho peor por parte de los participantes de la Universidad de la ciudad de Guadalajara de un $25.40 \%$ contra un $20.3 \%$ por parte de los participantes de la universidad en CDMX, mostrando que se ha visto más afectada la muestra bajo estudio en esta actividad con motivo de las restricciones generadas por la pandemia de Covid. Es decir, espacios abiertos como parques y jardines se encuentran cerrados, al igual que gimnasios y clubes.

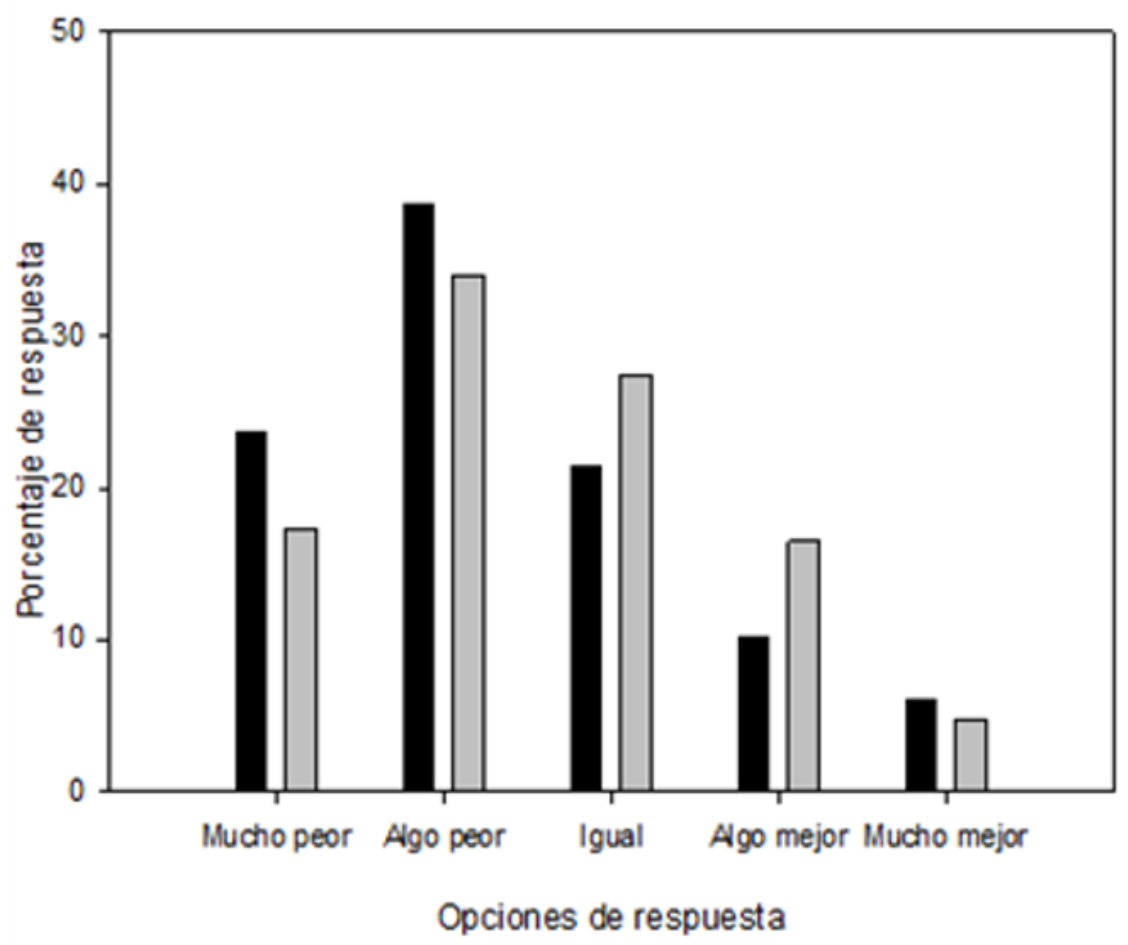

Fig.2.- Valoración de la calidad del sueño

Figura 2

En la Figura 2 con respecto a la calidad de sueño en el panel derecho superior predominan niveles superiores de valoración negativa en la muestra de la Universidad de la CDMX (62.2\% sumando las opciones mucho peor y algo peor) con respecto a la Universidad de la ciudad de Guadalajara (51.3\% sumando ambas categorías de respuesta), pero en ambos casos la valoración negativa es superior a la mitad de las muestras bajo estudio. 


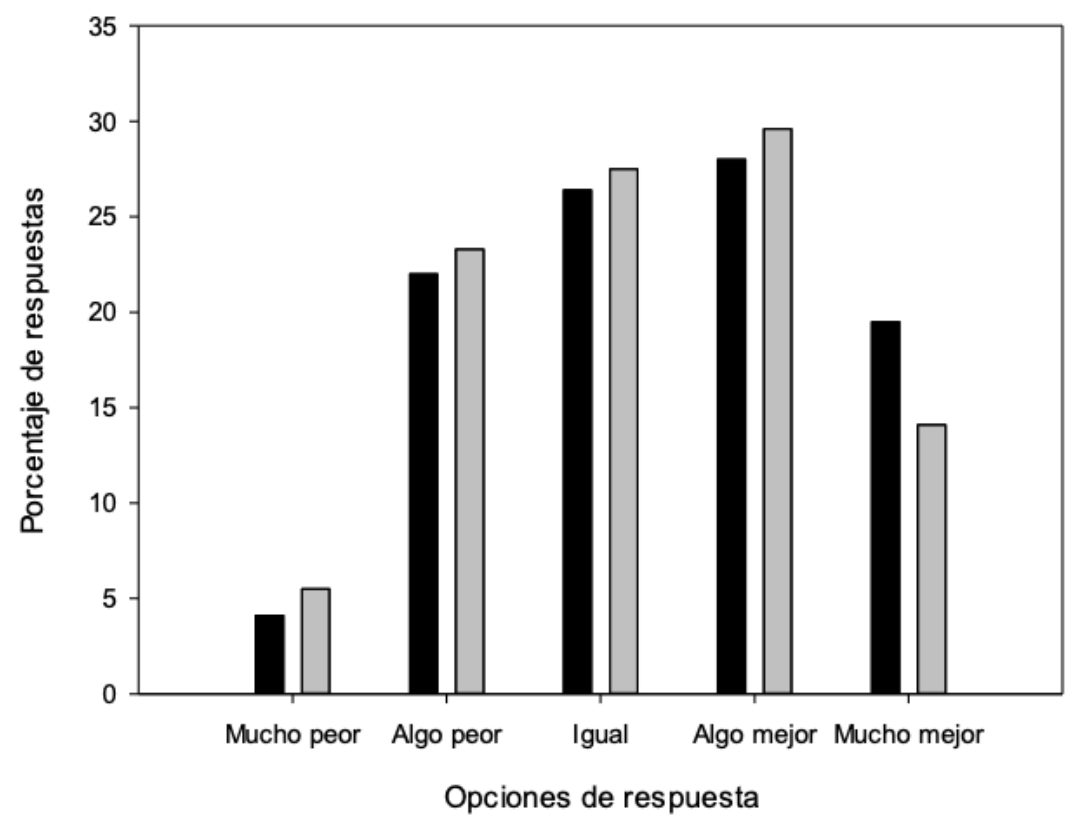

Fig. 3.- Valoración de la calidad de alimentación

Figura 3

En la Figura 3 se valora el efecto de la pandemia en la calidad de la alimentación por parte de ambas muestras. En la figura se observa una tendencia a hacer una valoración positiva durante la restricción de la pandemia. Un 4.1\% de la muestra de la Universidad en CDMX considera que es mucho peor la alimentación contra un 5.3\% de la muestra de la Universidad en Guadalajara. De forma consistente, es superior la valoración de algo peor con un $23.3 \%$ para esta Universidad contra un $22 \%$ de la Universidad de la CDMX. Como igual lo juzgan 27.5 de la muestra en Guadalajara contra un 26.4\% de la muestra de CDMX, por lo que se observa poca diferencia entre los porcentajes con una valoración mayor por parte de la muestra de universitarios en Guadalajara. 


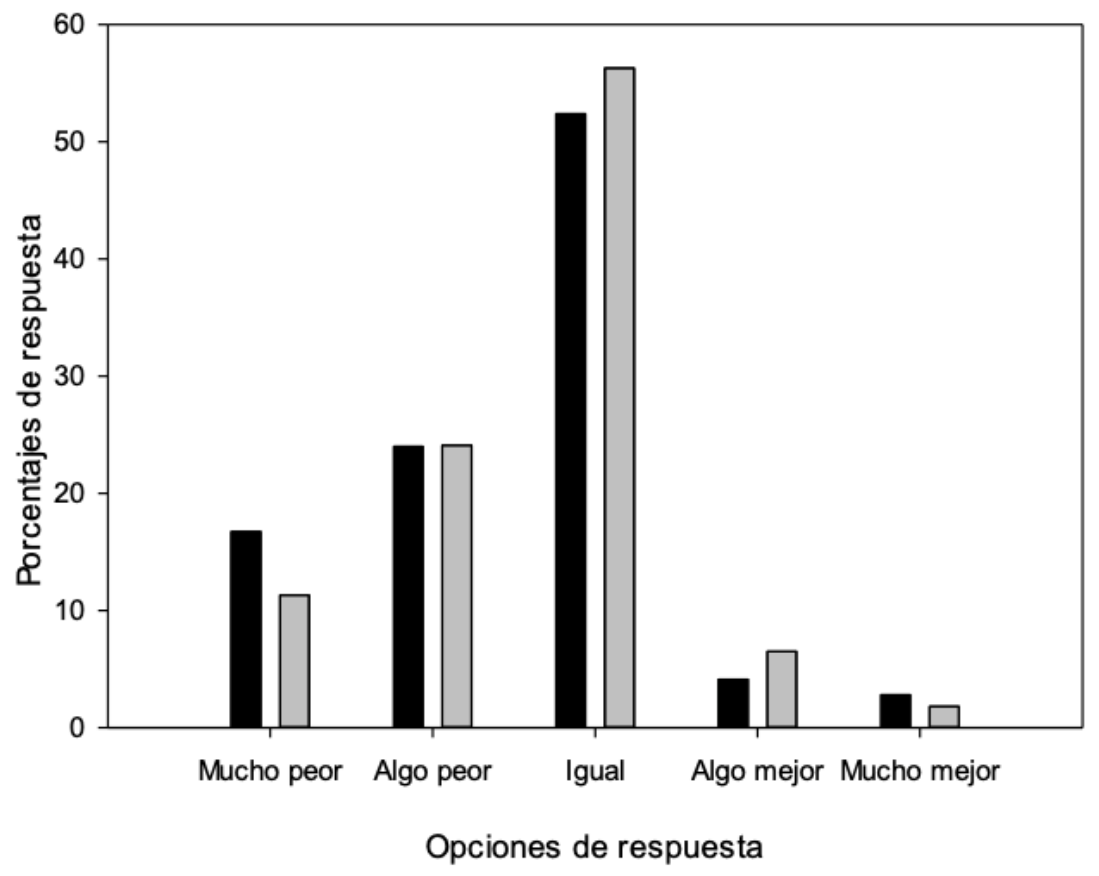

Fig. 4.- Valoración de ingresos económicos

Figura 4

En la Figura 4 se valora el efecto de la pandemia en el ingreso económico. Como puede observarse los porcentajes son muy semejantes entre ambas instituciones. Un $16.7 \%$ de la muestra de universitarios en CDMX lo valora como mucho peor y solamente un $11.3 \%$ en el caso de la otra universidad. Un $24 \%$ de ambas universidades consideran que algo peor. Solamente un 6.9\% de los participantes de CDMX considera que mejoró y un $8.3 \%$ en el caso de la muestra de la universidad en Guadalajara. En síntesis, un $40 \%$ valora un empeoramiento probablemente debido a la consideración de algún ingreso extra que ya no es posible recibir, pues ambas instituciones han mantenido los salarios establecidos como aparece con una mayor frecuencia la valoración de que se mantienen los ingresos económicos (52.4\% para muestra CDMX y $56.3 \%$ para muestra en Guadalajara). 


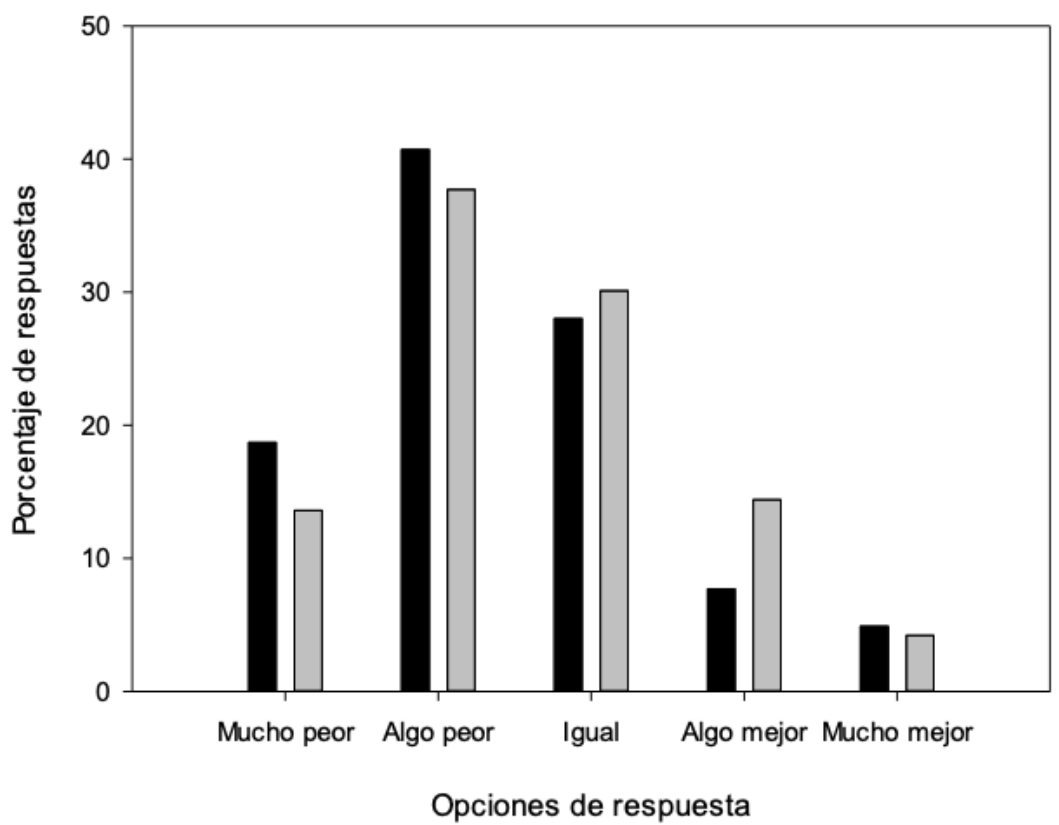

Fig. 5.- Valoración de actividad laboral

Figura 5

Muy relacionado con esto se encuentra la valoración de la actividad laboral durante la pandemia. En la Figura 5 se muestran los porcentajes de respuesta de las dos muestras estudiadas que son valoradas como peor por los participantes de CDMX (18.7\% mucho peor y $40.7 \%$ algo peor) contra lo reportado por los participantes en Guadalajara (13.6\% y $37.7 \%$ respectivamente). Más de la mitad valoran un empeoramiento de la actividad laboral. 


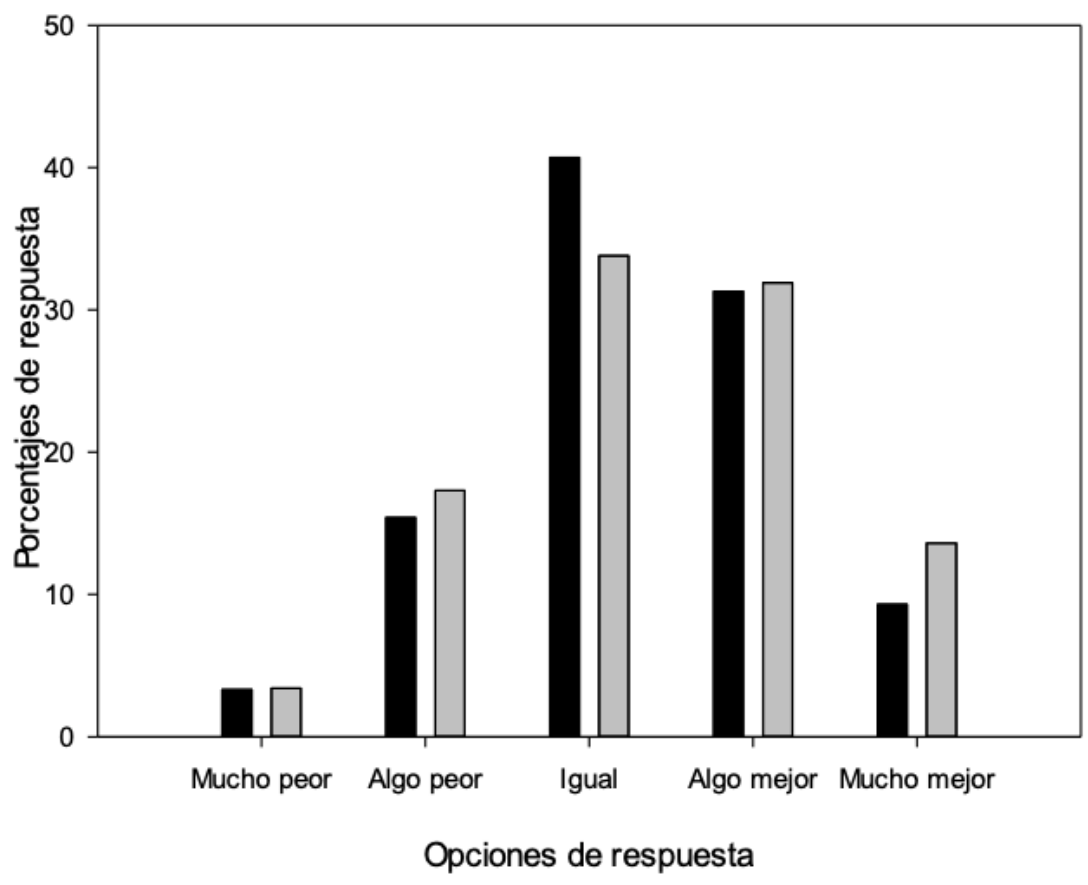

Fig.- 6.- Valoración de relaciones con pareja y familiares

Figura 6

Finalmente, se puede observar en la Figura 6 la valoración de empeoramiento en las relaciones de pareja y con familiares, el cual es supernior ligeramente en el caso de los participantes de la Universidad de la ciudad en Guadalajara ( $20.7 \%$ en la suma de mucho y algo peor), con respecto de los participantes de la Universidad de la CDMX (18.7\% en la suma de mucho y algo peor). Sin embargo, la mayoría de los participantes valoran que la calidad de las relaciones se mantiene o ha mejorado $(81.3 \%$ para CDMX vs $79.4 \%$ para la muestra de universitarios en Guadalajara). 


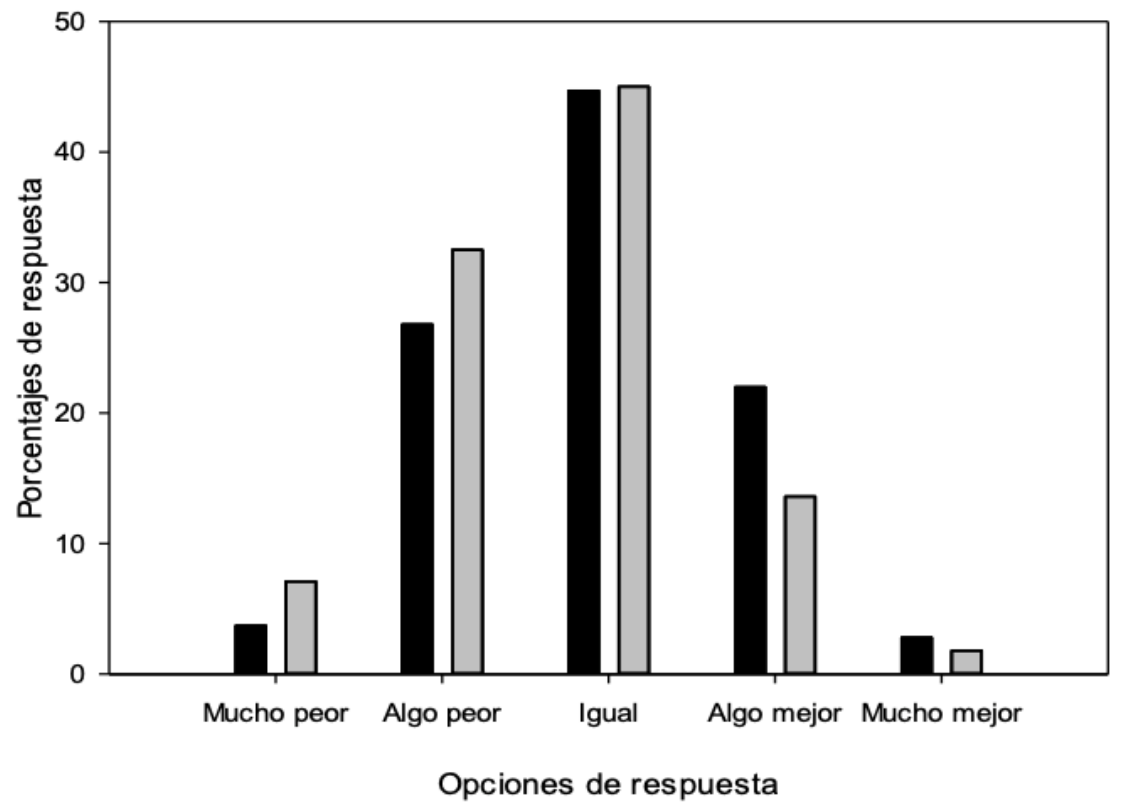

Fig. 7.- Valoración de relaciones con amigos durante la pandemia

Figura 7

Como se puede observar en el panel en la la Figura 7 se ilustra también, como en la figura anterior, una valoración de empeoramiento mayor en las relaciones con amigos por parte de los participantes de la Universidad de la ciudad de Guadalajara: $7.1 \%$ mucho peor y $32.5 \%$ algo peor con respecto de los participantes de la Universidad de la CDMX (barra obscura), con $3.7 \%$ mucho peor y $26.8 \%$ algo peor y en ambos casos siguen una distribución que se asemeja a lo normal. 


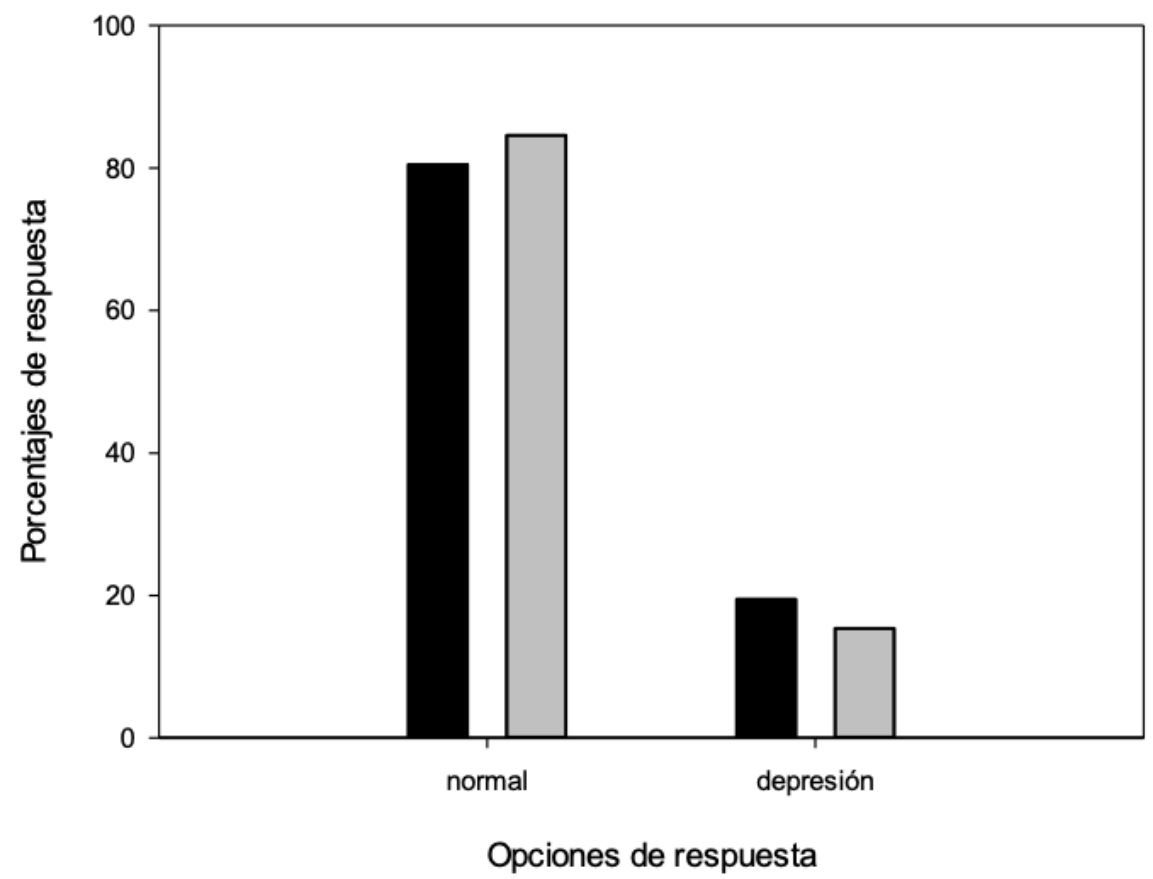

Figura 8.- Valoración de tener depresión durante pandemia

\section{Figura 8}

Con relación a la salud mental de los participantes tenemos los siguientes datos de valoración se muestra la Figura 8 en la que se graficó el porcentaje de las diferentes valoraciones de depresión durante la pandemia, las cuales son muy semejantes en las dos muestras analizadas, sin embargo, si existe una ligera superioridad en el porcentaje de casos de los participantes de la Universidad de la CDMX (19.5\%), que consideran tienen depresión y un menor porcentaje de la muestra de los universitarios en Guadalajara que se valoran como deprimidos (15.4\%). 


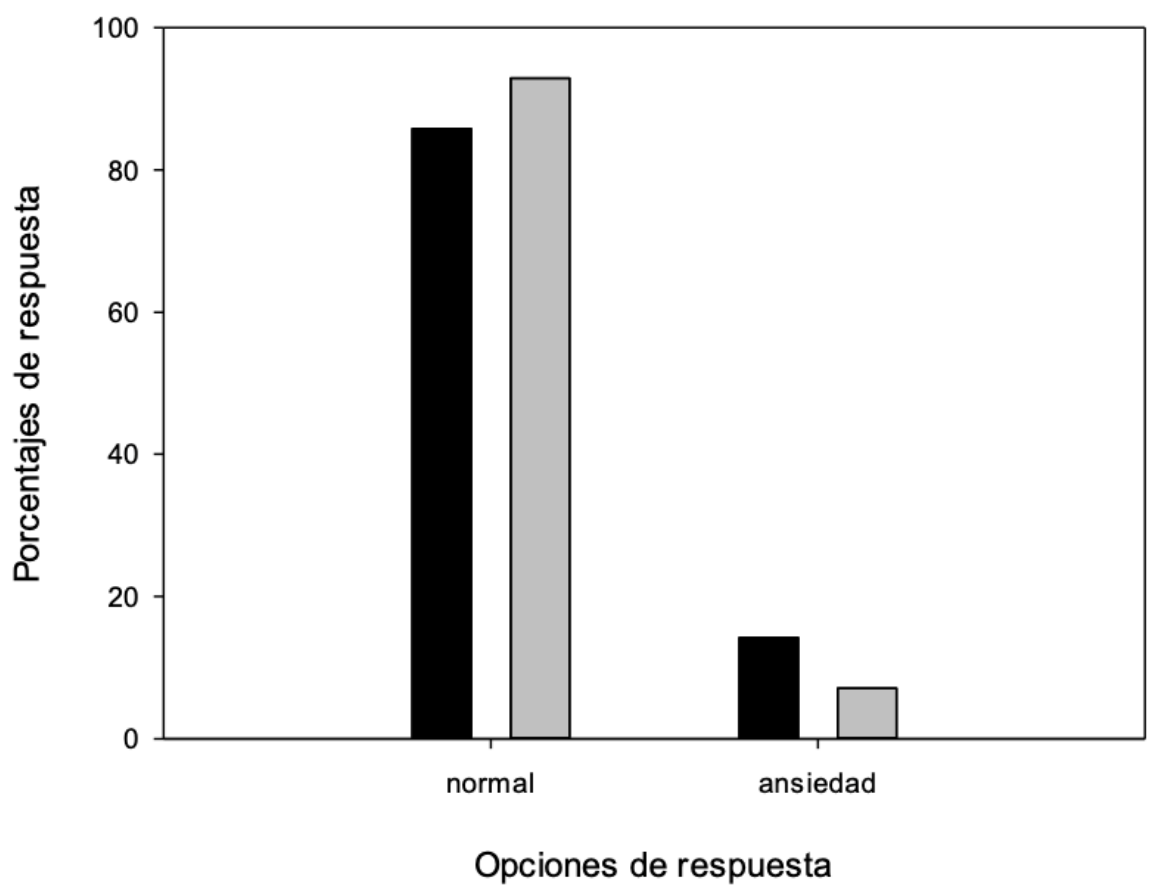

Figura 9.- Valoración de ansiedad durante la pandemia

\section{Figura 9}

Con respecto a la valoración de la ansiedad en la Figura 9 que muestra también que la valoración hecha por los participantes de la Universidad de la CDMX es el doble (14.2\%) con respecto de la realizada por los participantes de la Universidad de la ciudad de Guadalajara (7.1\%). 


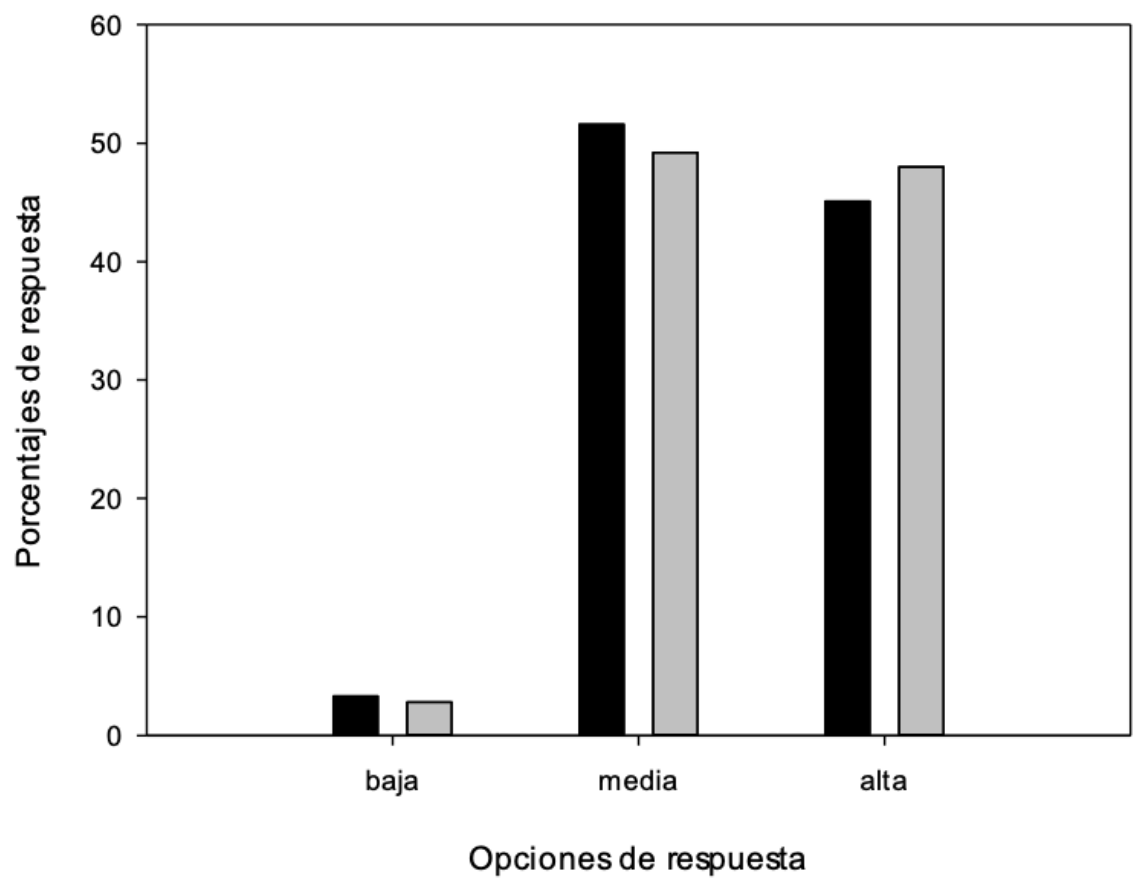

Fig. 10.- Valoración de resiliencia durante la pandemia

Figura 10

Con respecto a la resiliencia que se ilustra en la Figura 10 la valoración alta de la resiliencia durante la pandemia es superior en los participantes de la Universidad de la ciudad de Guadalajara (48\%), contra un $45.1 \%$ de los participantes de la CDMX. En el caso de la resiliencia media, la relación se invierte, siendo ligeramente superior en el caso de los participantes de la Universidad de la CDMX (51.6\%) contra un 49.2 de los participantes de la Universidad en Guadalajara. Destaca que la mayoría valora tener resiliencia media o alta para enfrentar la pandemia (96.7\% para CDMX y $97.2 \%$ para muestra en Guadalajara). 


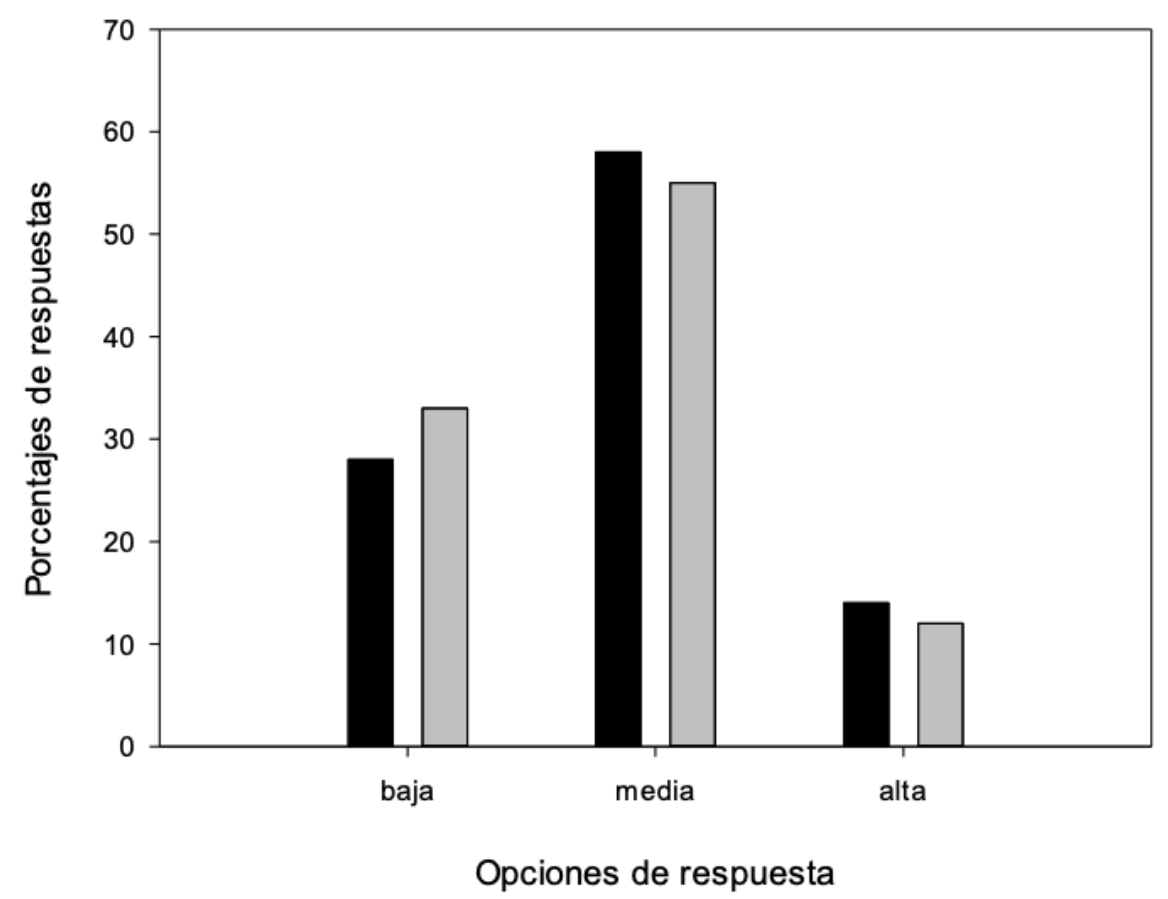

Fig. 11.- Valoración de crecimiento post-traumático durante la pandemia.

En la Figura 11 se observa una distribución semejante entre los participantes de ambas universidades. Sin embargo, la valoración de crecimiento post-traumático medio y alto es superior en los participantes de la Universidad de la CDMX (58\% y $14 \%$ respectivamente contra $55 \%$ y $12 \%$ para el caso de la muestra de universitarios en Guadalajara) y el crecimiento bajo de forma inversa es más alto entre los participantes de la Universidad de la ciudad de Guadalajara (33\% vs $28 \%$ para los universitarios de CDMX).

En la Tabla 2 se muestra el porcentaje de valoración de empeoramiento (mucho o poco) para la muestra total de ambas universidades con relación a las variables de hábitos de vida. 
Tabla 2

Tabla 2

Porcentaje de empeoramiento valorado por población general de las dos universidades en relación a distintos bábitos de vida

Variables Universidad en CDMX Universidad en Guadalajara

\begin{tabular}{lcc}
\hline Actividad Física & $55.7 \%$ & $59.2 \%$ \\
\hline Calidad del sueño & $62.2 \%$ & $51.3 \%$ \\
\hline
\end{tabular}

Calidad de alimentación

$26 \% \quad 28.8 \%$

Ingresos Económicos

$40.7 \% \quad 35.3 \%$

Actividad Laboral

$59.3 \%$

Los porcentajes muestran poca diferencia entre ambas muestras, siendo la mayor diferencia en calidad del sueño, valorándola peor la muestra de universitarios de la CDMX.

En la Tabla 3 se muestra el porcentaje de valoración de empeoramiento (mucho o poco) para la muestra total de ambas universidades con respecto a variables de adaptación y salud mental.

Tabla 3

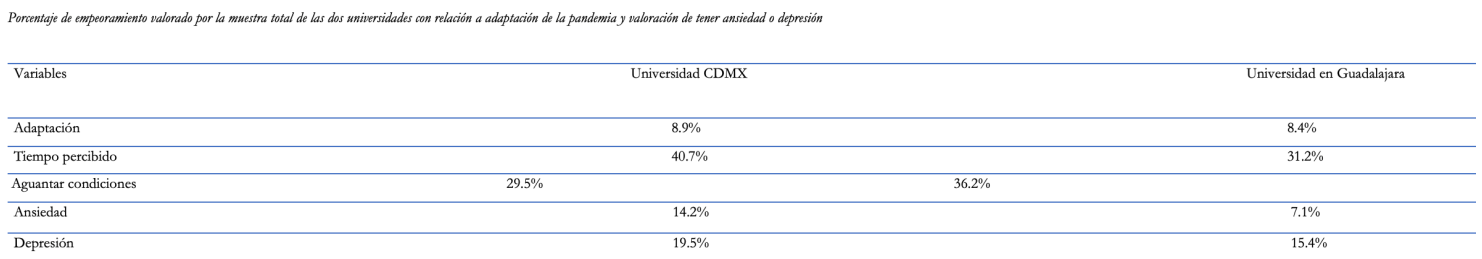

En los datos de ambas tablas se aplicó la prueba $\mathrm{T}$ y ninguna diferencia mostró ser significativa estadísticamente. Sin embargo, llama la atención que los universitarios de CDMX reporten el doble de porcentaje de empeoramiento en ansiedad y que también sean superiores en niveles de depresión. Más allá de las diferencias entre los grupos, resalta que el mayor porcentaje de ambos grupos valoran un empeoramiento respecto de actividad física, calidad de sueño y actividad laboral.

En relación a la variable de somatización el $72.8 \%$ de los universitarios de CDMX reportan dolor de cabeza contra un $65.4 \%$ de los participantes de la Universidad en Guadalajara, en relación a náuseas y dolor de 
estómago los porcentajes son de $58.1 \%$ y $37.4 \%$ respectivamente. En cuanto a falta de aire lo reporta un $22.8 \%$ de los participantes universitarios de la CDMX contra un porcentaje de $26.4 \%$ de parte de la universidad de Guadalajara. En relación a mareos no hay diferencias prácticamente pues unos reportan $24 \%$ y los otros $23.3 \%$. Finalmente, con relación a dolor de espalda los universitarios de CDMX tienen el 72.8\% y los de Guadalajara un 70\%. De forma que, a excepción de la falta de aire, con respecto a dolor de cabeza, mareos, náuseas y dolor de estómago, lo tuvieron de manera ligeramente superior los participantes universitarios de la CDMX en comparación con los porcentajes obtenidos por los participantes universitarios de Guadalajara. Los datos de somatización son consistentes con los datos de salud mental, pues en ambos casos existe una mayor prevalencia de empeoramiento entre los participantes de la CDMX.

$\mathrm{Al}$ respecto de la correlación entre las variables analizadas, se obtuvo comparando las opciones de respuesta de ambas muestras estudiadas, a continuación, se presentan los datos analizados desde esta perspectiva: 
Tabla 4

Tabla 4

Correlación entre variables de bábitos de vida en cada una de las muestras de las Universidades estudiadas

\begin{tabular}{|c|c|c|c|c|c|c|c|}
\hline & Muestras & $\begin{array}{l}\text { Calidad de } \\
\text { sueño }\end{array}$ & Alimentación & $\begin{array}{l}\text { Nivel de } \\
\text { Ingresos }\end{array}$ & $\begin{array}{c}\text { Actividad } \\
\text { laboral }\end{array}$ & $\begin{array}{l}\text { Relaciones } \\
\text { pareja y } \\
\text { familiares }\end{array}$ & $\begin{array}{c}\text { Relaciones } \\
\text { con } \\
\text { amigos }\end{array}$ \\
\hline \multirow{3}{*}{$\begin{array}{l}\text { Actividad } \\
\text { Física }\end{array}$} & U. de CDMX & $.241^{* *}$ & $.333^{* * *}$ & & & & \\
\hline & U..en Guad. & & & & & & \\
\hline & & $.359 * *$ & $.259^{* \text { *k }}$ & & & & \\
\hline \multirow{3}{*}{$\begin{array}{l}\text { Calidad de } \\
\text { sueño }\end{array}$} & U. de CDMX & & & & & & \\
\hline & ULen Gund & 1 & $.362^{\text {*ok }}$ & & & & \\
\hline & & & $.326^{* 0 *}$ & & & & \\
\hline Alimentac. & & & 1 & & & & \\
\hline $\begin{array}{l}\text { Nivel de } \\
\text { ingresos }\end{array}$ & & & & 1 & & & \\
\hline \multirow[t]{2}{*}{$\begin{array}{c}\text { Actividad } \\
\text { laboral }\end{array}$} & $\begin{array}{l}\text { U. de CDMX } \\
\text { U..en Guad. }\end{array}$ & & & $.394 * *$ & 1 & & \\
\hline & & & & $.374 * *$ & & & \\
\hline $\begin{array}{c}\text { Relaciones } \\
\text { con pareja y } \\
\text { familiares }\end{array}$ & & & & & & 1 & \\
\hline $\begin{array}{l}\text { Relaciones } \\
\text { con amigos }\end{array}$ & $\begin{array}{l}\text { U. de CDMX } \\
\text { U..en Guad. }\end{array}$ & & & & & $.222 * *$ & 1 \\
\hline & & & & & & $.168^{* *}$ & \\
\hline
\end{tabular}

$* *=p<0.001$

Como se puede observar las correlaciones son moderadas en ambas universidades y semejantes entre sí, pero significativas con una p de .001 como lo indica el doble asterisco en la tabla, lo que muestra la interrelación entre variables que atienden el mantenimiento y fortalecimiento corporal. La actividad física tiene correlaciones bajas pero significativas con alimentación y con calidad de sueño, lo que nos habla que los hábitos de vida vinculados al cuidado biológico están interrelacionados entre sí.

Asimismo, las correlaciones entre elementos del trabajo son altas entre sí y de menor nivel las relacionales. Las correlaciones son menores en la muestra de la Universidad en Guadalajara en comparación con la Universidad de la Capital.

A continuación, enlistaremos las correlaciones que se hicieron entre las diferentes variables evaluadas con respecto al campo de la salud mental para ambas universidades. Está claro como se observa en la Tabla 5 que la correlación entre depresión y ansiedad es de las más altas en ambas 
universidades, así como también la correlación entre la resiliencia y el crecimiento post-traumático.

Tabla 5

Correlación entre variables de salud mental en cada una de las muestras de las Universidades estudiadas

\begin{tabular}{cccccc}
\hline & Muestra & Depresión & Ansiedad & Resiliencia & $\begin{array}{c}\text { Crecimiento } \\
\text { Post-traumático }\end{array}$ \\
\hline Depresión & $\begin{array}{c}\text { Universidad } \\
\text { CDMX } \\
\text { Universidad en } \\
\text { Guadalajara }\end{array}$ & 1 & $.357^{\star \star}$ & $-.218^{\star \star}$ & $-.367^{\star \star}$ \\
\hline Ansiedad & $\begin{array}{c}\text { Universidad } \\
\text { CDMX } \\
\text { Universidad en } \\
\text { Guadalajara }\end{array}$ & $.391^{\star \star}$ & $-.277^{\star \star}$ & $-.238^{\star \star}$ \\
\hline Resiliencia & $\begin{array}{c}\text { Universidad } \\
\text { CDMX } \\
\text { Universidad en } \\
\text { Guadalajara }\end{array}$ & & $-.165^{\star \star}$ & $-.172^{\star \star}$ \\
& $\quad$ & & -.190 & $-.168^{\star \star}$ \\
\hline
\end{tabular}

Tabla 5

Con respecto a la edad es importante destacar que en la Universidad de la CDMX valoran peor los jóvenes la actividad física, el sueño, la alimentación, los ingresos económicos y la actividad laboral. Reportan también los jóvenes una mejora en las relaciones de pareja y familia, pero el empeoramiento prevalece mayor con los amigos y en los pasatiempos. El rango de edad de los jóvenes fue de los 16 a los 38 años. También este sector de edad de la muestra estudiada reporta una percepción de que el tiempo transcurre más lentamente con la pandemia. El 10.6\% reporta tener ansiedad y el 12.2\% depresión, porcentajes superiores con relación a otros segmentos de edad. En el caso de la Universidad en Guadalajara el segmento de edad media (de 38-59 años) son los que reportan un mayor empeoramiento con relación a actividad física, calidad del sueño y alimentación, así como los ingresos económicos. También reportan una mejoría en las relaciones familiares y de pareja y un empeoramiento en las relaciones con amigos y pasatiempos como en el caso de la otra universidad. En el segmento de los jóvenes coincide también en un mayor índice de ansiedad y depresión, así como una menor resiliencia y crecimiento post-traumático. Todas estas relaciones fueron significativas con la prueba $x^{2}$.

Con respecto de género destaca como diferencia significativa con $x^{2}$ una valoración peor del ingreso económico por parte de las mujeres en comparación con los hombres $x^{2}(8, \mathrm{~N}=382) 21.9333$ p menor que .005, así como también mayor ansiedad $x 2(8, \mathrm{~N}=382) 13.440$ con p menor que .001 y depresión $x^{2}(8, \mathrm{~N}=382) 11.396$ p menor que .003 en comparación con las respuestas de los hombres. De forma que las diferencias importantes son en el ingreso económico y en la ansiedad, y depresión con una valoración mayor por parte de las mujeres. 


\section{Discusión}

Los datos obtenidos en la presente investigación muestran más semejanzas que diferencias entre las muestras analizadas de las dos universidades y las diferencias obtenidas no fueron significativas estadísticamente, por lo que la hipótesis, de que contextos diferentes generan un impacto diferenciado no se comprueba. Una interpretación plausible, es que la variable educación (la cual es semejante entre universitarios de ambos contextos), genera un factor homogeneizador en las variables estudiadas de ambos grupos.

Una limitación importante es que, dado el tamaño de las muestras utilizadas, no son representativas de las poblaciones a las que pertenecen, por lo que su generalizabilidad se ve seriamente limitada. Sin embargo, la mayor valoración de ansiedad y depresión de la muestra de participantes de la universidad de la CDMX, pudiera explicarse en función de una mayor sobredemanda de estresores de diversos tipos en el contexto de una Ciudad de la magnitud y características como la CDMX. La hipótesis de diferencias por género y edad se ven confirmadas, mostrando un mayor impacto en las mujeres y en el sector joven.

Las correlaciones moderadas de ambas universitarios pero significativas entre las variables de salud (alimentación, sueño y actividad física), así como entre los elementos del trabajo (ingresos y calidad en el trabajo) ilustran la interdependencia que existe entre las variables evaluadas. De la misma manera, las correlaciones en las relaciones (con familiares y pareja y amigos y vecinos), que en su conjunto conforman un resultado particular en términos de calidad de vida y de bienestar subjetivo. Con respecto a la salud mental la interrelación entre ansiedad y depresión se confirma con las correlaciones obtenidas, así como en contraparte la correlación entre resiliencia y crecimiento post-traumático como relaciones directamente proporcionales. Asimismo, en el cruce de estas cuatro variables, las correlaciones negativas también confirman la hipótesis de interdependencia que existe entre las variables de salud mental medidas.

En consonancia con otros estudios, se confirman hallazgos realizados por otros investigadores en otros contextos distintos geográficamente y socioculturalmente, pero sometidos de la misma forma a la condición de la pandemia como se señaló previamente (Huang \& Zhao, 2020; Mazza et al., 2020; Sandín et al., 2020). El poder encontrar efectos semejantes en términos de ansiedad y depresión, ante una condición de amenaza común que ha quitado la vida a una gran cantidad de seres humanos, es un aspecto generalizado que vale destacar. Más allá de condiciones físicas diferentes, como clima, latitud y tipo de entorno, así como costumbres y patrones culturales distintos, el impacto psicosocial es semejante tanto en la salud mental como en aspectos cotidianos como los hábitos de vida vinculados al dormir, alimentarse, estar activo físicamente, trabajar, relacionarse con otros significativos como lo es la pareja, los familiares y los amigos. El mediador común para tener tales efectos comunes es el estrés producido por la pandemia, como reacción biológica común 
ante condiciones de amenaza (Selye, 1977), y condición para generar procesos de ansiedad y depresión (Camacho, 2015). Reconocer al estrés como elemento mediador común, además de reconocer teóricamente el proceso general de afectación tiene, la implicación práctica de desarrollar estrategias de manejo del estrés como formas de intervención psicológica preventiva (Robles Ortega \& Peralta Ramírez, 2010), promoviendo el autocuidado de la salud mediante el aprendizaje de hábitos de vida saludables (Camacho \& Vega, 2014), y aprendiendo también a ser resiliente ante condiciones de adversidad (Carver, 1998).

Es importante resaltar la mayor valoración de empeoramiento en el segmento de los más jóvenes que respondieron el cuestionario en ambas instituciones, así como también el reporte de mayor afectación en salud mental en el caso de las mujeres, en comparación con los hombres y con los adultos mayores respectivamente. Estos datos también son consistentes con otros estudios desarrollados (Ramírez \& Zerpa, 2020; Parrado-González \& León-Jariego, 2020), quienes también valoran un mayor empeoramiento en el caso de la actividad laboral lo cual sugiere una agenda de atención especial a estos casos particulares.

En el análisis comparativo entre universidades resalta la valoración más severa con relación a su salud mental, de los participantes de la Universidad de la CDMX, aunque como la diferencia no fue significativa no podemos concluir más allá de la diferencia encontrada en las muestras estudiadas. Como se ha mencionado arriba, suponemos un mayor estrés en la CDMX, generado por la alta concentración de personas y lo que conlleva de contaminación, tráfico y mayor inseguridad, pudiera explicar los mayores niveles de ansiedad y estrés valorados en los participantes de la Universidad situada en la CDMX. Junto con ello, la valoración de un mayor empeoramiento en la calidad del sueño, lo que probablemente implique un mayor nivel de alertamiento, siguiendo el modelo mencionado de Selye (1977) con el Síndrome de Alarma Generalizada. En la comparación de grupos el nivel de alertamiento es mayor al que es requerido por las personas de la Universidad en Guadalajara que viven en un contexto con las mismas problemáticas, pero en una magnitud mucho menor (Jusidman et al., 2016; Valadez Ramírez \& Bravo González, 2019). Este planteamiento requeriría el desarrollo de otra investigación para fundamentarlo con datos. Lo importante, es destacar que las dos muestras estudiadas reportaron valoración de empeoramiento en un alto porcentaje en varios de los hábitos de vida evaluados.

La mayor valoración de empeoramiento en la calidad de sueño en participantes de CDMX es consistente con la valoración de tener estados de ansiedad y depresión, más presentes en la muestra estudiada, en comparación con los participantes de la Universidad en Guadalajara como lo señala Villaseñor Lopez et al. (2020). La correlación entre ansiedad y depresión es de las más altas en ambas muestras, lo que confirman otros estudios que identifican una alta comorbilidad entre ambos aspectos de salud mental (Ramirez, et al., 2020). En el estudio referido se analiza el efecto del confinamiento por la pandemia en 
Venezuela en una muestra de 310 personas con Trastorno alimentario (105) y sin trastorno (205). Los resultados de un cuestionario reportan un $66.5 \%$ de la muestra con síntomas de estrés post-traumático y síntomas depresivos, lo cual implica un nivel alto.

Sin embargo, en población general, se identificó la presencia de ansiedad de $50 \%$ y prevalencia de depresión en $28 \%$, (Priego-Parra et al., 2020), lo cual implica un incremento con respecto a los porcentajes registrados previo a la presencia de las restricciones por la pandemia, mediante la aplicación de un cuestionario vía internet. Estos datos son superiores a los reportados por nuestras muestras analizadas. Se confirma como factores de riesgo ser joven, ser mujer y tener problemas de sueño para experimentar ansiedad y depresión. Un 25.3\% experimentó ambas cosas y los porcentajes incrementaron de forma significativa, comparado con un estudio control previo a la presencia de la pandemia.

Es probable que la muestra de universitarios tenga más recursos de afrontamiento que la población general para lidiar con estos estresores generados por la pandemia, como señala Díaz et al., (2020). En este estudio desarrollado con 343 estudiantes universitarios españoles destaca como recursos del estudiantado: la resiliencia, el capital social online que se tiene como forma de comunicación durante la pandemia y las alternativas para obtener satisfacción vital a pesar de las circunstancias generadas por la pandemia. Se aplicaron varias escalas para evaluar las variables mencionadas en estudiantes de licenciatura con un $80 \%$ de mujeres.

Con respecto a somatización cuando menos en un día de la pandemia más de la mitad de la muestra estudiada (52.69\%) han tenido problemas de salud física de los participantes de CDMX, contra un 44.5\% de la muestra de universitarios de Guadalajara. Esto es consistente también con los datos de salud reportados por Galindo et al. (2020), en una investigación realizada con población abierta mediante un cuestionario aplicado entre marzo y abril del 2020 a 1508 participantes. En este estudio se reporta que $31 \%$ analiza continuamente sus sensaciones corporales y los interpretaba como síntomas de enfermedad y $15 \%$ con temor a contraer enfermedad. De igual forma, tener antecedentes de alguna comorbilidad física o mental es un factor de riesgo para experimentar ansiedad o depresión severa. Al igual que ser mujer, joven, soltero y sin hijos (estos dos últimos factores por el aislamiento social que implica).

En cuanto a la valoración del ingreso es muy semejante entre ambas universidades probablemente debido a que siguen políticas semejantes de sostenimiento de salarios y además en ambos casos se considera que un $30 \%$ percibe un empeoramiento, probablemente debido a ingresos extras que no han podido ser realizados debido a las políticas de restricción en casa y al desplome del consumo en consecuencia.

A manera de conclusión se puede señalar que las restricciones de la pandemia han tenido un impacto en la población universitaria compuesta por docentes, estudiantes y empleados administrativos, en niveles ligeramente menores que los reportados a nivel mundial y nacional (Berenzon et al., 2013; Bueno-Notivol et al., 2021). La formación 
educativa tanto de los estudiantes como de los docentes los proveen de mayores recursos de afrontamiento para poder lidiar con la pandemia.

El hecho de poder desarrollar trabajo a distancia en casa, al menos en el caso de docentes y estudiantes ha tenido implicaciones de mejoría en aspectos como la alimentación y las relaciones con la pareja y la familia que han sido más continuas y cercanas para la mayoría de la muestra estudiada. En otros hábitos como la actividad física, el sueño, la valoración del trabajo que implica hacerlo a distancia y las relaciones con los amigos han empeorado de forma importante para los participantes en el estudio.

En términos teóricos, una limitación importante del presente estudio es que es muy relevante considerar las condiciones anteriores al inicio de la pandemia para evaluar la real afectación, y dentro de estas condiciones tanto el contexto físico como el sociocultural, desgraciadamente no tenemos mediciones de los mismos participantes antes de las condiciones de la pandemia, aunque estudios como el de Priego-Parra et al. (2020) si, como lo hemos referido líneas arriba. De la misma forma, es relevante enfatizar que lo que analizamos son relaciones entre el comportamiento verbal de respuesta a diferentes preguntas que conformaron el cuestionario y dicho comportamiento puede tener mayor o menor correspondencia con las situaciones reales dependiendo de variaciones individuales. En términos metodológicos se vuelve importante establecer la diferencia que existe entre el decir y el hacer de los miembros de las muestras estudiadas y su grado de correspondencia (Luciano, 1993).

En el campo de la aplicación se considera relevante, el procurar recuperar los niveles de hábitos de vida que tenían las personas estudiadas, previo a la aparición de la pandemia, como un recurso de afrontamiento para mejorar la salud mental, mediante estrategias integrales que involucren a los grupos familiares y desarrollar estrategias de satisfacción vital que no impliquen riesgo de contagio.

Como hemos mencionado arriba, nuestra muestra no permite generalizar los hallazgos a la población de las universidades de las muestras estudiadas, sin embargo, otros estudios referidos en el presente trabajo, con muestras más significativas y en población abierta, han coincidido en las relaciones encontradas en nuestro estudio, a manera de una validación convergente. Los datos obtenidos como un primer estudio exploratorio, es sugerente para corroborar con muestras mayores las relaciones encontradas, en el reconocimiento de que, en este momento, nos encontramos en otro estado de la pandemia. Es importante identificar los factores de riesgo en población universitaria para que sea adecuadamente atendida, ya que la potencial protección de tener un buen nivel educativo, no exime de los riesgos de afectación psicológicos expresados en los hábitos de vida y la salud mental de los universitarios. La relevancia del presente estudio, es analizar a una población específica que muestra sus potenciales vulnerabilidades para que sean consideradas en la política pública en salud. 


\section{Agradecimientos}

Los autores agradecen el apoyo en el análisis de datos de Ana Lucía Camacho, Paola Bátiz y Luis Hernando Silva.

\section{Referencias}

Almagiá, E. B. (2004). Apoyo social, estrés y salud. Psicología y Salud, 14(2), 237-243.

Ammar, A., Mueller, P., Trabelsi, K., Chtourou, H., Boukhris, O., Masmoudi, L., ... \& ECLB-COVID19 Consortium. (2020). Psychological consequences of COVID-19 home confinement: The ECLB-COVID19 multicenter study. PloS One, 15(11), e0240204. https://doi.org/10.1371 /journal.pone.0240204

Berenzon, S., Lara, M. A., Robles, R., \& Medina-Mora, M. E. (2013). Depresión: estado del conocimiento y la necesidad de políticas públicas y planes de acción en México. Salud Pública de México, 55(1), 74-80.

Bueno-Notivol, J., Gracia-García, P., Olaya, B., Lasheras, I., López-Antón, R., \& Santabárbara, J. (2021). Prevalence of depression during the COVID-19 outbreak: A meta-analysis of community-based studies. International Journal of Clinical and Health Psychology, 21(1), 100196. https://doi.org /10.1016/j.ijchp.2020.07.007

Cálad, C. A. A. (2003). Los vínculos afectivos y la estructura social. Una reflexión sobre la convivencia desde la red de promoción del buen trato. Investigación \& Desarrollo, 11(1), 70-103.

Camacho, E., \& Vega, C. (2014). Autocuidado de la salud. ITESO.

Camacho, E. (2015). Estrés, personalidad y depresión: algunos indicadores para actuar de manera preventiva. En E. Camacho \& S. Galán (Eds.), Alternativas psicológicas de intervención en problemas de salud ( $\left.1^{\mathrm{a}} \mathrm{ed}\right)$ (pp. 171-182). Manual Moderno.

Carver, C. S. (1998). Resilience and thriving: Issues, models, and linkages. Journal of Social Issues, 54(2), 245-266. https://doi.org/10.1111/j.15404560.1998.tb01217.x

\section{Referencias}

Chandu, V. C., Marella, Y., Panga, G. S., Pachava, S., \& Vadapalli, V. (2020). Measuring the impact of COVID-19 on mental health: A scoping review of the existing scales. Indian Journal of Psychological Medicine, 42(5), 421-427. https://doi.org/10.1177/0253717620946439

Di Renzo, L., Gualtieri, P., Pivari, F., Soldati, L., Attinà, A., Cinelli, G., ... \& De Lorenzo, A. (2020). Eating habits and lifestyle changes during COVID-19 lockdown: An Italian survey. Journal of Translational Medicine, 18, 229. https://doi.org/10.1186/s12967-020-02399-5

Díaz, A. L., Prados, J. S. F., Canos, V. F., \& Martínez, A. M. M. (2020). Impactos del confinamiento por el COVID-19 entre universitarios: satisfacción vital, resiliencia y capital social online. RISE, 9(1), 79-104. 
Ferrer, L. P. (2020). El COVID 19: impacto psicológico en los seres humanos. Revista Arbitrada Interdisciplinaria de Ciencias de la Salud. SALUD Y VIDA, 4(7), 188-199.

Galindo, O., Ramírez, M., Costas, R., Mendoza, L., Calderillo, G., \& Meneses, A. (2020). Síntomas de ansiedad, depresión y conductas de autocuidado durante la pandemia de Covid-19 en la población general. Gaceta Médica de México, 156(4) 298-305.

Gobierno de México. (2021). Todo sobre el COVID-19. https// coronavirus.gob.mx

Guerrero-Zúñiga, S., Gaona-Pineda, E. B., Cuevas-Nasu, L., Torre-Bouscoulet, L., Reyes-Zúñiga, M., Shamah-Levy, T., \& Pérez-Padilla, R. (2018). Prevalencia de síntomas de sueño y riesgo de apnea obstructiva del sueño en México. Salud Publica de Mexico, 60(3), 347-355. https://doi.org/10 $.21149 / 9280$

Huang, Y., \& Zhao, N. (2020). Generalized anxiety disorder, depressive symptoms and sleep quality during COVID-19 outbreak in China: A webbased cross-sectional survey. Psychiatry Research, 288, 112954. https://do i.org/10.1016/j.psychres.2020.112954

Jusidman, C., Camas, F. J., Carreón, I. G., \& Marín, O. E. (2016). El crecimiento urbano y las violencias en México. Naciones Unidas. http://www.cepal.org /es/publicaciones/39899-crecimiento-urbano-violencias-mexico

Kroenke, K., Spitzer, R. L., \& Williams, J. B. (2003). The Patient Health Questionnaire-2: Validity of a two-item depression screener. Medical Care, 41(11), 1284-1292. http://dx.doi.org/10.1097/01.MLR.0000093 487.78664.3C

Kroenke, K., Spitzer, R. L., Williams, J. B., Monahan, P. O., \& Löwe, B. (2007). Anxiety disorders in primary care: Prevalence, impairment, comorbidity, and detection. Annals of Internal Medicine, 146(5), 317-325. https://doi .org/10.7326/0003-4819-146-5-200703060-00004

López Fernández, D., \& Sanz, A. (2020). ¿Nos deja secuelas el confinamiento? The Conversation. https://accedacris.ulpgc.es/bitstream/10553/75725/2 /31.\%20\%C2\%BFNos\%20deja\%20secuelas\%20el\%20confinamiento_.p df

Löwe, B., Wahl, I., Rose, M., Spitzer, C., Glaesmer, H., Wingenfeld, K., ... \& Brähler, E. (2010). A 4-item measure of depression and anxiety: Validation and standardization of the Patient Health Questionnaire-4 (PHQ-4) in the general population. Journal of Affective Disorders, 122(1-2), 86-95. ht tps://doi.org/10.1016/j.jad.2009.06.019

Luciano, M. C. (1993). La conducta verbal a la luz de recientes investigaciones. Su papel sobre otras conductas verbales y no verbales. Psicothema, 5(2), 351-374.

Martínez Gómez, J. A., Bolívar Suárez, Y., Yanelli Yanez-Peñúñuri, L., \& Rey Anacona, C. A. (2020). Tendencias de la investigación sobre síntomas de trastornos mentales durante la pandemia por COVID-19. Medicina UPB, 39(2), 24-33. https://doi.org/10.18566/medupb.v39n2.a05

Mazza, C., Ricci, E., Biondi, S., Colasanti, M., Ferracuti, S., Napoli, C., \& Roma, P. (2020). A nationwide survey of psychological distress among Italian people during the COVID-19 pandemic: Immediate psychological responses and associated Factors. International Journal of Environmental 
Research and Public Health, 17(9), 3165. https://doi.org/10.3390/ijerph 17093165

Marquina Lujan, R. J. (2020). Autopercepción del estrés en aislamiento social en tiempos de covid -19. Revista ConCiencia EPG, 5(1), 83 - 97. https:// doi.org/10.32654/CONCIENCIAEPG.5-1.6

Medina-Ortiz, O., Araque-Castellanos, F., Ruiz-Domínguez, L. C., RiañoGarzón, M., \& Bermudez, V. (2021). Trastornos del sueño a consecuencia de la pandemia por COVID-19. Revista Peruana de Medicina Experimental y Salud Pública, 37(4), 755-761. https://doi.org/ 10.17843/rpmesp.2020.374.6360

Moreno-Proaño, G. (2020). Pensamientos distorsionados y ansiedad generalizada en COVID-19. CienciAmérica, 9(2), 251-255. http://dx.do i.org/10.33210/ca.v9i2.314

Niederkrotenthaler, T., Gunnell, D., Arensman, E., Pirkis, J., Appleby, L., Hawton, K., John, A., Kapur, N., Khan, M., O'Connor, R. C., \& Platt, S. (2020). Suicide research, prevention, and COVID-19: Towards a global response and the establishment of an international research collaboration. Crisis, 41(5), 321-330. https://doi.org/10.1027/0227-5910/a000731

Organización Mundial de la Salud (OMS). (1948). Constitución de la Organización Mundial de la Salud. https://www.who.int/es/about/gove rnance/constitution

Organización Mundial de la Salud (OMS). (2021). Preguntas $y$ respuestas sobre la enfermedad por coronavirus (COVID-19). https://www.who.int/es/emergencies/diseases/novel-coro navirus-2019/advice-for-public/q-a-coronaviruses

Organización Panamericana de la Salud. (2020). La OMS caracteriza a COVID-19 como una pandemia. https://www.paho.org/es/noticias/11-3 -2020-oms-caracteriza-covid-19-como-pandemia

Parrado-González, A., \& León-Jariego, J. C. (2020). COVID-19: factores asociados al malestar emocional y morbilidad psíquica en población española. Revista Española de Salud Pública, 94(8), 1-16.

Pérez-Rodrigo, C., Citores, M. G., Hervás Bárbara, G., Litago, F. R., Casis Sáenz, L., Aranceta-Bartrina, J., ... \& Quiles, J. (2020). Cambios en los hábitos alimentarios durante el periodo de confinamiento por la pandemia COVID-19 en España. Revista Española de Nutrición Comunitaria, 26(2), 28010. https://doi.org/10.14642/RENC.2020.26.2.5213

Priego-Parra, B. A., Triana-Romero, A., Pinto-Gálvez, S. M., Ramos, C. D., Salas-Nolasco, O., Reyes, M. M., Ramos-de-la-Medina, A., \& RemesTroche, J. M. (2020). Anxiety, depression, attitudes, and internet addiction during the initial phase of the 2019 coronavirus disease (COVID-19) epidemic: A cross-sectional study in México. medRxiv. htt ps://doi.org/10.1101/2020.05.10.20095844

Proto, E., \& Quintana-Domeque, C. (2021). COVID-19 and mental health deterioration by ethnicity and gender in the UK. PloS one, 16(1), e0244419. https://doi.org/10.1371/journal.pone.0244419

Ramírez-Ortiz, J., Castro-Quintero, D., Lerma-Córdoba, C., Yela-Ceballos, F., \& Escobar-Córdoba, F. (2020). Mental health consequences of the COVID-19 pandemic associated with social isolation. Colombian Journal of Anesthesiology / Revista Colombiana de Anestesiología, 48(4), 1-7. http s://doi.org/10.5554/22562087.e930 
Ramírez, A., \& Zerpa, C. E. (2020). Relaciones entre confinamiento domiciliario por la pandemia COVID-19: hábitos y estado emocional en personas con o sin conductas sugestivas de Trastornos del comportamiento alimentario residentes de zonas urbanas de Venezuela. Boletin Cientifico Sapiens Research, 10(2), 15-23.

Riaz, M., Abid, M., \& Bano, Z. (2021). Psychological problems in general population during covid-19 pandemic in Pakistan: Role of cognitive emotion regulation. Annals of Medicine, 53(1), 189-196. https://doi.org/ $10.1080 / 07853890.2020 .1853216$

Robles Ortega, H., \& Peralta Ramírez, M. I. (2010). Programa para el control del estrés (2a ed.). Ediciones Pirámide.

Sandín, B., Valiente, R. M., García-Escalera, J., \& Chorot, P. (2020). Impacto psicológico de la pandemia de COVID-19: efectos negativos y positivos en población española asociados al periodo de confinamiento nacional. Revista de Psicopatología y Psicología Clínica, 25(1), 1-22. https://doi.org /10.5944/rppc.27569

Segerstrom, S. C., \& Miller, G. E. (2004). Psychological stress and the human immune system: A meta-analytic study of 30 years of inquiry. Psychological Bulletin, 130(4), 601-630. https://doi.org/10.1037/0033-2909.130.4.60 1

Selye, H. (1977). The stress of my life. A scientist's memoirs. McClelland and Stewart.

Valadez Ramírez, A. A., \& Bravo González, M. C. (2019). Estresores urbanos, estrés y afrontamiento en habitantes de la Ciudad de México. Revista Electrónica de Psicología Iztacala, 22(4), 2965-2982.

Villaseñor Lopez, K., Jimenez Garduño, A. M., Ortega Regules, A. E., Islas Romero, L. M., Gonzalez Martinez, O. A., \& Silva Pereira, T. S. (2020). Cambios en el estilo de vida y nutrición durante el confinamiento por SARS-CoV-2 (COVID-19) en México: un estudio observacional. Revista Española De Nutrición Humana Y Dietética, 25(Supl. 2), e1099. https:// doi.org/10.14306/renhyd.25.S2.1099

Vivanco Vidal, A., Saroli Araníbar, D., Caycho Rodríguez, T., Carbajal León, C., \& Noé Grijalva, M. (2020). Ansiedad por Covid-19 y salud mental en estudiantes universitarios. Revista de Investigación en Psicologia, 23(2), 197-216. https://doi.org/10.15381/rinvp.v23i2.19241

Zijlema, W. L., Stolk, R. P., Löwe, B., Rief, W., White, P. D., \& Rosmalen, J. G. (2013). How to assess common somatic symptoms in large-scale studies: A systematic review of questionnaires. Journal of Psychosomatic Research, 74(6), 459-468. https://doi.org/10.1016/j.jpsychores.2013.03.093 